\title{
Corpus spongiosum abscess presented as new-onset diabetes in an adult patient: a case presentation
}

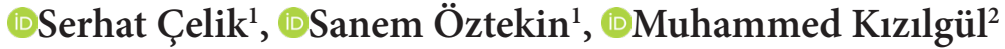 \\ ${ }^{1}$ Department of Internal Medicine, University of Health Sciences, Diskapi Training and Research Hospital, Ankara, Turkey \\ ${ }^{2}$ Department of Endocrinology and Metabolism, University of Health Sciences, Diskapi Training and Research Hospital, Ankara, Turkey
}

Cite this article as: Çelik S, Öztekin S, Kızılgül M. Corpus spongiosum abscess presented as new-onset diabetes in an adult patient: a case presentation. Anatolian Curr Med J 2021; 3(1): 69-71.

\begin{abstract}
Rare infections, known as signal infections might be pathognomonic for patients with diabetes mellitus. A 55-year-old man without a significant medical history was admitted to our hospital with polyuria, polydipsia, dysuria, fever, chills and weight loss for the last month. A laboratory investigation showed leukocytosis and, elevated levels of C-reactive protein, sedimentation rate, blood glucose, and HbAlc. The patient was hospitalized in the internal medicine service and started intensive insulin therapy with intravenous saline infusion. The patient's fever and chills were not improved despite ceftriaxone treatment for three days. Ceftriaxone-resistant, imipenem-sensitive E. coli was grown in the blood cultures, so ceftriaxone was stopped and imipenem plus cilastatin combination was started. Detailed physical examination of the patient for fever etiology showed severe swelling in the perineal region. Superficial and scrotal ultrasonography and then pelvic magnetic resonance imaging revealed corpus spongiosum abscess. The perineal region was punctured and numerous Gram-negative bacilli and polymorphonuclear leukocytes were seen in the gram stain. Drainage catheter was inserted into the corpus spongiosum. Blood sugar levels were regulated and the patient was discharged after the antibiotic treatment was completed. As in our case, signal infections should be kept in mind especially in patients admitted with new onset of diabetes mellitus and persistent fever. A detailed physical examination should be performed in these patients and atypical areas like perineum should be carefully examined.
\end{abstract}

Keywords: Corpus spongiosum abscess, new-onset diabetes, signal infection

\section{INTRODUCTION}

Diabetes mellitus $(\mathrm{DM})$ is characterized by the deficiency of insulin secretion or action (1). The prevalence of diabetes is increasing as a result of rising obesity prevalence as population aging. Diabetic patients have increased morbidity and mortality, especially from cardiovascular and renal complications. As a result of decreased defense mechanisms, negative effects of hyperglycemia and obesity, neuropathy and impaired tissue perfusion, gastrointestinal and urinary dysmotility, moderate or severe infection-related morbidities are more common in patients with diabetes (2). Diabetics have an increased risk for lower respiratory tract and urinary tract infections and, cellulitis. 3 Signal infections are rare and severe infections that are not self-limiting and likely to be diagnosed regardless of differences in physician behavior or previous diabetes diagnosis. They are often pathognomonic of a patient with diabetes mellitus (4). Emphysematous pyelonephritis, malignant otitis externa, endophthalmitis, mucormycosis, and Fournier's gangrene are all examples of signal infections (5).
We herein report a case of corpus spongiosum abscess as a rare infection and new onset of diabetes mellitus in a patient presented with fever, chills and weight loss.

\section{CASE REPORT}

A55-year-old man without a significant medical history was admitted to our hospital with polyuria, polydipsia, dysuria, fever, chills and weight loss for the last month. Nausea and vomiting have also been added to the current complaints within the last 3 days. No abnormal findings were found except decreased skin turgor at the physical examination. A laboratory investigation showed leukocytosis (13.300/ $\mu \mathrm{L})$ and, elevated level of C-reactive protein $(114 \mathrm{mg} /$ $\mathrm{dl})$, sedimentation (20 mm/hour), blood glucose (446 $\mathrm{mg} / \mathrm{dl}$ ) and HbAlc (12.1\%). Pyuria, bacteriuria as well as ketonuria were seen in urine examination. There was no acidosis on blood gas analysis. The patient was hospitalized in the internal medicine service and started 
intensive insulin therapy with intravenous (IV) saline infusion. Blood sugar was followed and basal-bolus insulin therapy was administered. Because of fever, blood and urine culture were taken and ceftriaxone was started. However, the patient's fever and chills were not improved despite treatment for three days. Urine culture result was negative. Ceftriaxone-resistant, imipenem-sensitive E.coli was grown in the blood cultures, so ceftriaxone was stopped and imipenem plus cilastatin combination were added to the treatment.

Detailed physical examination of the patient for fever etiology showed severe swelling with palpation in the perineal region. Superficial and scrotal ultrasonography of the patient revealed an irregular hypoechoicheterogeneous collection area consistent with abscess, approximately $50 \times 30 \mathrm{~mm}$ in size, in the perineal region of the corpus spongiosum. The corpus spongiosum was heterogeneous in appearance and its thickness was increased. A pelvic magnetic resonance imaging (MRI) revealed a collection extends from the right lobe of prostate to corpus spongiosum and penis, measures approximately $40 \mathrm{~mm}$ in the thickest area, with septa, circumferential enhancing after IV contrast medium, showing diffuse restriction in diffusion-weighted sequences, which is consistent with the abscess (Figure). The perineal region was punctured and numerous gram-negative bacilli and polymorphonuclear leukocytes were seen in the gram stain. Abscess culture was growing E.coli susceptible to antibiotics that the patient has been already used.

Drainage catheter was inserted into the corpus spongiosum of the patient by an interventional radiologist.
The patient's drain was followed daily and the drain was removed when there was no return. The patient's blood sugar levels regulated and discharged after the antibiotic treatment was completed.

The patient had good glycemic control and normal biochemical values after six months of follow-up and no abscess was detected in the control MRI.

\section{DISCUSSION}

Diabetes mellitus leads to several complications such as vascular diseases and renal failure, which eventually affect the overall survival of patients. As a general concept, diabetics are generally more susceptible to infections (2). Despite this common belief, there are very few investigations which have conclusively evaluated the overall risk for infections in patients with DM. Shah et al. (6) observed a higher rate of bacterial infections such as osteomyelitis, pyelonephritis and cystitis, pneumonia, cellulitis, sepsis or peritonitis in diabetics. Another study reported an increased susceptibility to lower respiratory tract, urinary tract, and bacterial skin and mucous membrane infections in this population (7). A higher risk for wound infections in diabetics may be related to a higher number of leg ulcers in these patients. Some rare infections such as emphysematous pyelonephritis, invasive otitis externa, emphysematous cholecystitis or rhinocerebral mucormycosis are more common in patients with DM. Moreover, infections caused by Staphylococcus aureus or Mycobacterium tuberculosis are also more prevalent in these patients.

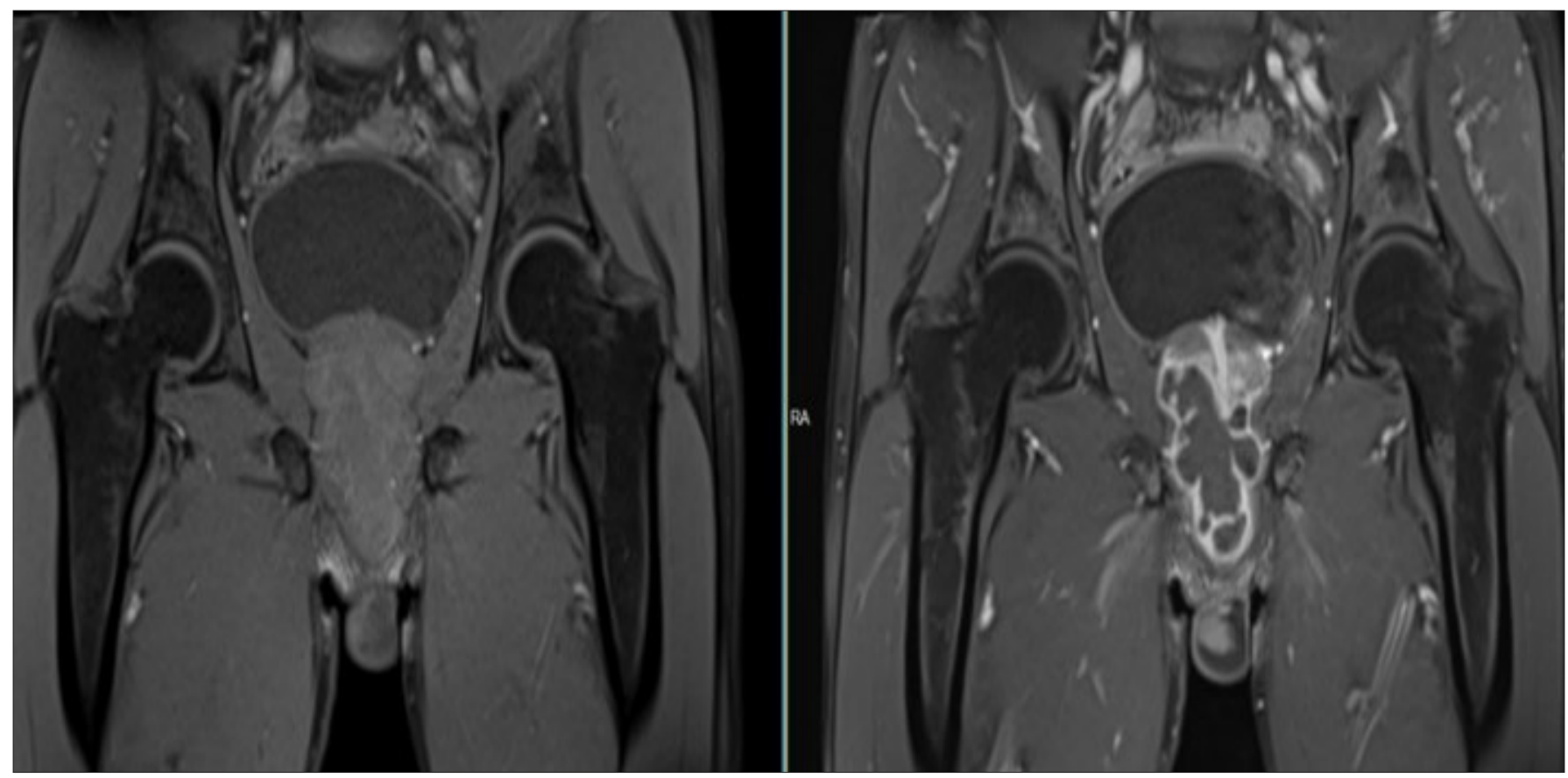

Figure. A pelvic magnetic resonance imaging (MRI) revealed a collection extends from the right lobe of the prostate to corpus spongiosum and penis, measures approximately $40 \mathrm{~mm}$ in the thickest area, with septa, circumferential enhancing after IV contrast medium, showing diffuse restriction in diffusion-weighted sequences, which is consistent with the abscess 
There are several mechanisms of infection-induced diabetes including direct destruction of beta cells (e.g. parotitic pancreatitis), molecular mimicry as microbial antigens share homologies with host antigens (e.g. Cytomegalovirus and Epstein-Barr virus), autoimmunity targeted to beta cells as a result of increased processing and presentation of autoantigens during infection or epitope spreading, increased inflammation and release of cytokines, increased insulin requirement and insulin resistance during infection. Moreover, poor glycemic control may impair humoral and cellular immunity that may lead to a decrease in neutrophil chemotaxis, neutrophil adherence to vascular endothelium, intracellular bactericidal activity, phagocytosis, and opsonization (8-11). Glycation decreases the expression of class I major histocompatibility complex on the surface of myeloid cells, which leads to impaired cellular immunity (12).

Infections may impair glycemic control or DM can be presented with an infection9. Signal infections including malignant external otitis, rhinocerebral mucormycosis, and gangrenous cholecystitis almost always seen in patients with DM. Abscess of the corpus spongiosum is also an example of signal infection. Corpus spongiosum of the penis is a mass of erectile tissue that lies along the lower side of the penis and is located below the pair of corpus cavernosum, and it contains $90 \%$ of the blood volume throughout normal erection. Abscess of the corpus spongiosum is quite rare. Only one case of corpus spongiosum abscess reported by Kubata $\mathrm{M}$ et al. (13) in the literature. To our knowledge, our case is the only case of corpus spongiosum abscess as a signal infection lead to impaired glycemic control in a diabetic patient.

\section{CONCLUSION}

As in our case, signal infections should be kept in mind especially in patients admitted with new onset of diabetes mellitus and persistent fever. A detailed physical examination should be performed in these patients and atypical areas like perineum should be carefully examined.

\section{ETHICAL DECLARATIONS}

Informed Consent: Written informed consent was obtained from all participants who participated in this study.

Referee Evaluation Process: Externally peer-reviewed. Conflict of Interest Statement: The authors have no conflicts of interest to declare.

Conflict of Interest Statement: The authors have no conflicts of interest to declare.

Financial Disclosure: The authors declared that this study has received no financial support.
Author Contributions: All of the authors declare that they have all participated in the design, execution, and analysis of the paper, and that they have approved the final version.

\section{REFERENCES}

1. Casqueiro J, Casqueiro J, Alves C. Infections in patients with diabetes mellitus: A review of pathogenesis. Indian J Endocrinol Metab 2012; 16: 27-36.

2. Bertoni, Alain G., Sharon Saydah, and Frederick L. Brancati. Diabetes and the risk of infection-related mortality in the US. Diabetes Care 2001; 24: 1044-9.

3. Muller LM, Gorter KJ, Hak E, et al. Increased risk of common infections in patients with type 1 and type 2 diabetes mellitus. Clin Infect Dis 2005; 41: 281-8.

4. Cockram CS, Lee N. Diabetes and Infections. In: Holt R, Cockram C, Flyvbjerg A, Goldstein B, eds. Textbook of Diabetes, 4th ed. Chichester: Wiley-Blackwell, 2010.

5. Pearson-Stuttard J, Blundell S, Harris T, Cook DG, Critchley J. Diabetes and infection: assessing the association with glycaemic control in population-based studies. Lancet Diabetes Endocrinol 2016; 4: 148-58.

6. Shah BR, Hux JE: Quantifying the risk of infectious diseases for people with diabetes. Diabetes Care 2003; 26: 510-13

7. Muller LM, Gorter KJ, Hak E, Goudzwaard WL, Schellevis FG, Hoepelman AI, Rutten GE: Increased risk of common infections in patients with type 1 and type 2 diabetes mellitus. Clin Infect Dis 2005; 41: 281-8

8. Saeed NK, Al-Biltagi M. Diabetes and Infections: Which is the Fuel?. 2014.

9. Cooke FJ. Infections in people with diabetes. Medicine 2015; 43: 41-3.

10. Geerlings SE, Hoepelman AI. Immune dysfunction in patients with diabetes mellitus (DM). FEMS Immunol Med Microbiol 1999; 26: 256-65.

11. Muller LM, Gorter KJ, Hak E, et al. Increased risk of common infections in patients with type 1 and type 2 diabetes mellitus. Clin Infect Dis 2005; 41: 281-8.

12. Price CL, Al Hassi HO, English NR, Blakemore AI, Stagg AJ, Knight SC. Methylglyoxal modulates immune responses: relevance to diabetes. J Cell Mol Med 2010; 14: 1806-15.

13. Kubota M, Kanno T, Nishiyama R, Okada T, Higashi Y, Yamada $\mathrm{H}$. A case of abscess of corpus spongiosum associated with rectal cancer. Hinyokika Kiyo 2013; 59: 539-43. 